\title{
Protocatechuic acid benefits proliferation and phenotypic maintenance of rabbit articular chondrocytes: An in vitro study
}

\author{
LIKE LUO $^{1,2^{*}}$, QINGJUN WEI ${ }^{1,2^{*}}$, LEI LIU $^{1,2}$, XIAO LIN $^{3,4}$, CUIWU LIN $^{3}$, LI ZHENG $^{2,5}$ and JINMIN ZHAO ${ }^{1,2}$ \\ ${ }^{1}$ Department of Orthopedic Trauma and Hand Surgery, The First Affiliated Hospital; \\ ${ }^{2}$ Guangxi Key Laboratory of Regenerative Medicine, Guangxi Medical University, Nanning, Guangxi 530021; \\ ${ }^{3}$ School of Chemistry and Chemical Engineering, Guangxi University, Nanning, Guangxi 530004; \\ ${ }^{4}$ Guangxi Key Laboratory of Traditional Chinese Medicine Quality Standards, \\ Guangxi Institute of Traditional Medical and Pharmaceutical Sciences, Nanning, Guangxi 530022; \\ ${ }^{5}$ The Medical and Scientific Research Center, Guangxi Medical University, Nanning, Guangxi 530021, P.R. China
}

Received November 5, 2014; Accepted February 6, 2015

DOI: $10.3892 /$ etm.2015.2326

\begin{abstract}
Numerous antioxidants exhibit antiarthritic effects due to their inhibitory effect on inflammatory factors. Certain antioxidants, such as protocatechuic acid (PCA) and its analogs, have been reported to be effective in the treatment of arthritis. However, the effect of PCA on chondro-protection may be alleviated due to the induction of apoptosis, as has been demonstrated in stomatocytes. To clearly determine the effect of PCA on the biological and cellular metabolism of rabbit articular chondrocytes in vitro, examinations of cytotoxicity, proliferation and morphology were performed, in addition to analyses of glycosaminoglycan (GAG) synthesis and the expression of cartilage-specific genes. The results revealed that PCA effectively promoted chondrocyte growth, the synthesis of the extracellular matrix and the mRNA expression of aggrecan, collagen II and Sox9, while downregulating the expression of the collagen I gene, a marker of chondrocyte dedifferentiation. In addition, hypertrophy, which may result in chondrocyte ossification, was not detected in the groups. Among the doses (range, $0.05-0.3 \mathrm{mmol} / \mathrm{l}$ ) of PCA that promoted the proliferation of chondrocytes, a concentration of $0.125 \mathrm{mmol} / \mathrm{l}$ produced the optimum performance. The results indicated that PCA, particularly at a dose of $0.125 \mathrm{mmol} / 1$,
\end{abstract}

Correspondence to: Ms. Li Zheng, The Medical and Scientific Research Center, Guangxi Medical University, 22 Shuangyong Road, Nanning, Guangxi 530021, P.R. China

E-mail: zhengli224@163.com

*Contributed equally

Abbreviations: PCA, protocatechuic acid; OA, osteoarthritis; ECM, extracellular matrix; GAG, glycosaminoglycan; HA, hyaluronate acid; HE, hematoxylin-eosin; RT-qPCR, reverse transcription-quantitative polymerase chain reaction

Key words: protocatechuic acid, polyphenols, prochondrogenic agent, rabbit articular chondrocyte, dedifferentiation, proliferation accelerated the proliferation of rabbit articular chondrocytes in vitro and maintained their phenotype. This study may provide a basis for further research concerning the treatment of cartilage defects.

\section{Introduction}

As a tissue that possesses a poor regenerative capacity, articular cartilage tends to be structurally broken down or degenerated under the shadow of disease, aging or trauma, finally evolving into osteoarthritis (OA), where the prognosis is poor $(1,2)$. Over the course of OA development, catabolic factors, including proinflammatory cytokines, are activated, which induces the gradual self-destruction of cartilage coupled with the curb of chondrogenic differentiation (3-5). Accompanied with this process is the impact of non-cartilage-specific extracellular matrix (ECM) with inferior mechanical properties, which is produced by dedifferentiated chondrocytes and can prevent chondroprogenitors from remodeling cartilage defects through migration $(6,7)$. These factors lead to the acceleration and deterioration of OA.

As a traditional anti-inflammatory agent, non-steroidal anti-inflammatory drugs have been administered to patients with OA to attenuate associated symptoms, similar to other therapies, including aminoglucose and sodium hyaluronate (HA) (8). Tissue engineering, where cells can be encapsulated in a carrier matrix, is a promising alternative resolution although arthroplasty is time consuming and microfracture suitable for minor lesions (9). Sustained phenotypic maintenance of chondrocytes during expansion in vitro, and the synthesis of mature ECM combined with anti-inflammation following transplantation in vivo, are necessary for the successful treatment of OA with tissue engineering $(10,11)$, which remains a serious challenge and requires further research.

With marked antioxidation, anti-inflammatory and antitumor properties, polyphenolic compounds isolated from plants, such as green tea, and catechins, are attracting increasing attention. Evidence suggests that polyphenols aid the differentiation and phenotypic survival of numerous stomatocytes (12). Among these, protocatechuic acid (PCA) 
has been reported to possess analgesic and anti-inflammatory activity in Freund's adjuvant arthritis (13). In addition, PCA has been shown to stimulate the apoptosis of tumor cells (14) and resist the HA degradation of experimental arthritis (15). An additional study demonstrated that PCA benefited cell differentiation and the maintenance of cellular phenotypes for neural stem and progenitor cells in vitro (16). These findings indicated that as a potent anti-inflammatory agent, PCA may exert an effect on chondrocyte differentiation, which is of significance for the treatment of long-term arthritis, via the maturation of ECM secretion or the induction of chondrocyte expansion in cartilage tissue engineering.

Therefore, PCA was hypothesized to be a potential chondro-protective agent that may be applied to induce chondrocyte in vitro expansion in cartilage tissue engineering for OA. In the present study, the effect of PCA on the biological functions of rabbit articular chondrocytes in vitro were investigated through the determination of cytotoxicity, proliferation and cell morphology, coupled with glycosaminoglycan (GAG) synthesis and cartilage-specific gene expression. This study may provide a reference for the application of PCA in cartilage tissue engineering and the treatment of OA.

\section{Materials and methods}

Isolation and culture of articular chondrocytes. A total of five one-week-old New Zealand rabbits were purchased from the Center of Experimental Animals of Guangxi Medical University (Nanning, China) and the relative operations were approval by the Ethics Committee of Guangxi Medical University. First, one rabbit was anaesthetized using pentobarbitone sodium $(60 \mathrm{mg} / \mathrm{kg}$, intraperitoneally; Sigma-Aldrich, St. Louis, MO, USA). After sedation had been successfully induced, $150 \mathrm{mg}$ pentobarbitone sodium was slowly administered in addition to the initial dose, until the rabbit was euthanized. Standard preoperative preparation was performed and cartilage slices were harvested from hip and knee joints on a clean bench (JB-CJ-2FX; Suzhou Jiebao Purification Engineering Equipment Co., Ltd., Jiangsu, China). These slices were primarily dissociated with $0.25 \%$ trypsin (Solarbio, Beijing, China) for $30 \mathrm{~min}$, and then with $2 \mathrm{mg} / \mathrm{ml}$ collagenase type II (Sigma-Aldrich) in $\alpha$-modified Eagle's medium ( $\alpha$-MEM; Gibco Life Technologies, Carlsbad, CA, USA) for $3 \mathrm{~h}$. Chondrocytes were isolated through centrifugation $\left(400 \times \mathrm{g}, 5 \mathrm{~min}, 37^{\circ} \mathrm{C}\right.$ ) and resuspended in $\alpha$-MEM containing $20 \%(\mathrm{v} / \mathrm{v})$ fetal bovine serum (Gibco Life Technologies) and $1 \%(\mathrm{v} / \mathrm{v})$ antibiotics (penicillin $100 \mathrm{U} / \mathrm{ml}$ and streptomycin $100 \mathrm{U} / \mathrm{ml}$; Solarbio). The cultures were maintained in a 5\% $\mathrm{CO}_{2}$ incubator (Thermo Fisher Scientific, Glasgow, UK) at $37^{\circ} \mathrm{C}$, with the culture medium changed every three days. Cells were passaged after reaching $80-90 \%$ confluence. Confluent chondrocytes in a logarithmic growth phase were prepared for the further experiments.

Preparation and treatment of PCA. PCA was purchased from Chengdu Must Bio-technology Co., Ltd. (Chengdu, China). Prior to the experiment, PCA was dissolved in $75 \%$ alcohol to form a $100-\mathrm{mmol} / \mathrm{l}$ stock solution. Following filtration with a $0.22-\mu \mathrm{m}$ filter (EMD Millipore, Billerica, MA, USA) for sterilization, the solution was stored at $-4^{\circ} \mathrm{C}$. The stock solu- tion of PCA was added to the cell culture to provide various concentrations for the subsequent experiments.

Cytotoxicity assay. Articular chondrocytes were cultured in 96-well microplates (Corning Incorporated, New York, NY, USA) pretreated with various concentrations of PCA (0-1 mmol/l) for three days. MTT $(5 \mathrm{mg} / \mathrm{ml}$; Gibco Life Technologies) was added to the cultures in each well. Following incubation at $37^{\circ} \mathrm{C}$ for $4 \mathrm{~h}$, the culture medium was removed and dimethyl sulfoxide (Gibco Life Technologies) was added (150 $\mu \mathrm{l}$ per well). The microplates were gently shaken for 10 min by MH-2 Mini Shaker (Kylin-Bell Lab Instruments Co., Ltd., Jiangsu, China) in order to obtain a completely dissolved purple solution. The optical density was detected at $570 \mathrm{~nm}$ using a Multiskan GO Microplate spectrophotometer (Thermo Fisher Scientific, USA).

Cell proliferation analysis and biochemical assay. In light of the results of the cytotoxicity assay, three doses of PCA with evident positive effects were selected $(0.0625,0.125$ and $0.25 \mathrm{mmol} / \mathrm{l})$, along with a control group ( $0 \mathrm{mmol} / 1 \mathrm{PCA})$, for the cell proliferation analysis and biochemical assay. Chondrocytes in the different groups were cultured for two, four and six days. The cells were digested with $0.25 \%$ trypsin and resuspended in phosphate-buffered saline (PBS) containing $60 \mu \mathrm{g} / \mathrm{ml}$ proteinase $\mathrm{K}$ (Sigma-Aldrich) for $16 \mathrm{~h}$ at $60^{\circ} \mathrm{C}$. Following the application of Hoechst 33258 (Sigma-Aldrich), the proliferation of cells was analyzed via DNA production using an ultraviolet Hitachi F-4500 spectrofluorometer (Hitachi, Ltd., Tokyo, Japan) at $460 \mathrm{~nm}$, with the absorbance value of Hoechst 33258 dye used as the baseline.

The total production of GAGs was measured through absorbance with a 1,9-dimethylmethylene blue spectrophotometric assay at $525 \mathrm{~nm}$, with chondroitin sulfate (Sigma-Aldrich) as the standard sample. The synthesis and secretion of GAGs was calculated according to the standard curve and normalized against the total DNA production, which revealed the biosynthetic activity of the cells in diverse culture media.

Morphological examination. Following culture for six days, the cells were fixed with $95 \%$ alcohol for $30 \mathrm{~min}$ and then rinsed three times in PBS, once every 3 min. Subsequently, chondrocytes were stained by hematoxylin-eosin (HE) using a commercial kit (Jiancheng Biotech Co., Ltd., Nanjing, China) according to the manufacturer instructions. An inverted phase contrast microscope (Zeiss International, Oberkochen, Germany) was used to conduct the cell morphological analysis.

Reverse transcription-quantitative polymerase chain reaction (RT-qPCR) analysis. To further investigate the effect of PCA on the expression of cartilage-specific genes, the mRNA expression levels of collagen I, II and X, aggrecan and Sox 9 were analyzed by RT-qPCR. Total RNA was extracted from the articular chondrocytes using an RNeasy RNA extraction kit (Tiangen Biotechnology, Beijing, China), according to the manufacturer's instructions. Total RNA ( 300 ng) was used as a template and reverse transcribed into cDNA with a reverse transcription kit (Fermentas; Thermo Fisher Scientific). cDNA was amplified using a SYBR ${ }^{\circledR}$ Green Master Mix kit 
Table I. Primer sequences used in the reverse transcription-quantitative polymerase chain reaction experiments.

\begin{tabular}{lll}
\hline mRNA & \multicolumn{1}{c}{ Forward primer } & Reverse primer \\
\hline GAPDH & 5'-CTATAAATTGAGCCCGCAGC-3' & 5'-ACCAAATCCGTTGACTCCG-3' \\
Aggrecan & 5'-CTACACGCTACACCCTCGAC-3' & 5'-ACGTCCTCACACCAGGAAAC-3' \\
Type I collagen & 5'-GTTCAGCTTTGTGGACCTCCG-3' & 5'-GCAGTTCTTGGTCTCGTCAC-3' \\
Type II collagen & 5'-AAGCTGGTGAGAAGGGACTG-3' & 5'-GGAAACCTCGTTCACCCCTG-3' \\
Type X collagen & 5'-CGCTGAACGATACCAAATGCC-3' & 5'-TTCCCTACAGCTGATGGTCC-3' \\
Sox9 & 5'-AAGCTCTGGAGACTTCTGAACG-3' & 5'-CGTTCTTCACCGACTTCCTCC-3'
\end{tabular}

GAPDH, glyceraldehyde 3-phosphate dehydrogenase.

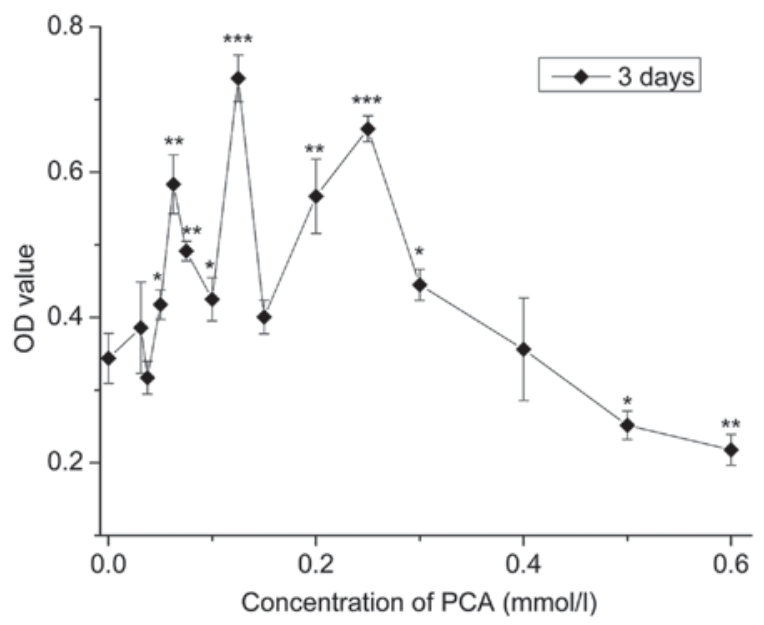

Figure 1. Cytotoxicity analysis of the chondrocytes treated with different concentrations of PCA after three days. Results are expressed as the mean \pm standard deviation $(\mathrm{n}=4) .{ }^{*} \mathrm{P}<0.05,{ }^{* *} \mathrm{P}<0.01$ and ${ }^{* * *} \mathrm{P}<0.001$ vs. control group ( $0.0 \mathrm{mmol} / 1 \mathrm{PCA})$. PCA, protocatechuic acid; OD, optical density.

(Roche Diagnostics GmbH, Mannheim, Germany). qPCR was performed using a qPCR Detection System (Realplex 4; Eppendorf, Enfield, CT, USA) with FastStart Universal $\mathrm{SYBR}^{\circledR}$ Green Master Mix at $95^{\circ} \mathrm{C}$ for $5 \mathrm{~min}$ for the initial denaturation, then 40 cycles of $95^{\circ} \mathrm{C}$ for $15 \mathrm{~s}$ and $60^{\circ} \mathrm{C}$ for 1 min. The designed primers used for PCR are shown in Table I. The primer specificity was confirmed by analyzing the dissociation curve of each primer pair. Relative gene expression levels were calculated using the $2^{-\Delta \Delta C t}$ method, relative to GAPDH gene expression. Each gene was analyzed in triplicate to reduce randomization error.

Statistical analysis. Statistical analyses were conducted using SPSS software, version 17.0 (SPSS, Inc., Chicago, IL, USA). Results are expressed as the mean \pm standard deviation for quantitative data. Statistical significance was determined using one way analysis of variance followed by Dunnett's post hoc test. $\mathrm{P}<0.05$ was considered to indicate a statistically significant difference.

\section{Results}

Cytotoxicity assay. As depicted in Fig. 1, compared with the control group (0 mmol/l), 0.03125-0.4 mmol/1 PCA indicated
A

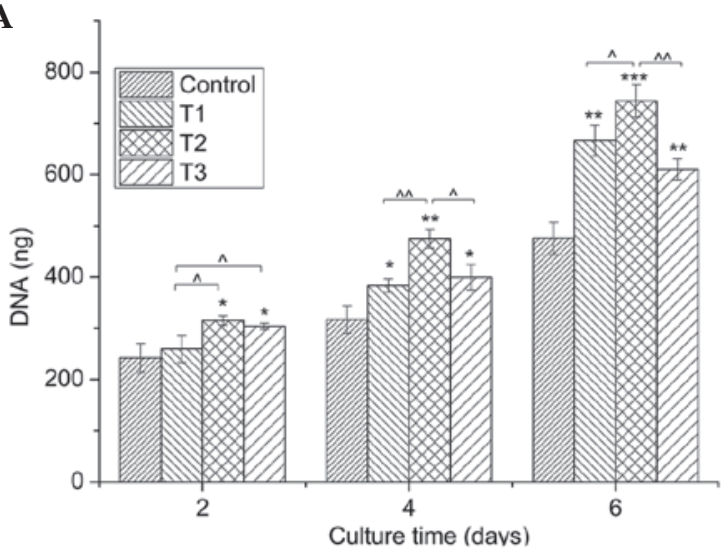

B

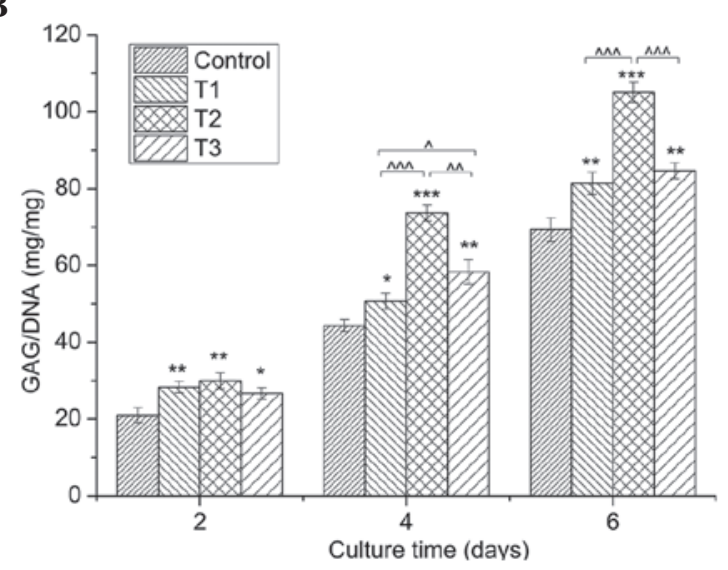

Figure 2. Quantification of cell proliferation by the detection of DNA content and matrix production by GAG analysis. (A) Proliferation of chondrocytes cultured in vitro with 0 (control), 0.0625 (T1), 0.125 (T2) and $0.25 \mathrm{mmol} / 1$ (T3) protocatechuic acid for two, four and six days. (B) GAG synthesis (mg) normalized against DNA content (mg). Data from three independent experiments were evaluated, and the results are presented as the mean \pm standard deviation. ${ }^{* \wedge} \mathrm{P}<0.05 ;{ }^{* * \wedge} \mathrm{P}<0.01$; and ${ }^{* * *, \wedge \wedge} \mathrm{P}<0.001$. ${ }^{*},{ }^{* *}$ and ${ }^{* * *}$ vs. control. ${ }^{\wedge},{ }^{\wedge \wedge}$ and ${ }^{\wedge \wedge \wedge}$ vs. as indicated. GAG, glycosaminoglycan.

OD values with no statistical significance or higher that indicated low cytotoxicity; OD values of $0.0625-0.3 \mathrm{mmol} / 1 \mathrm{PCA}$ significantly increased $(\mathrm{P}<0.05)$, indicating $0.0625-0.3 \mathrm{mmol} / 1$ PCA accelerated cell growth $(\mathrm{P}<0.05)$, with the most evident effect at a dose of $0.125 \mathrm{mmol} / \mathrm{l}$. By contrast, at concentrations ranging between 0.5 and $0.6 \mathrm{mmol} / 1 \mathrm{PCA}$, proliferation inhibition of the rabbit articular chondrocytes in vitro was observed when compared with the control group. 


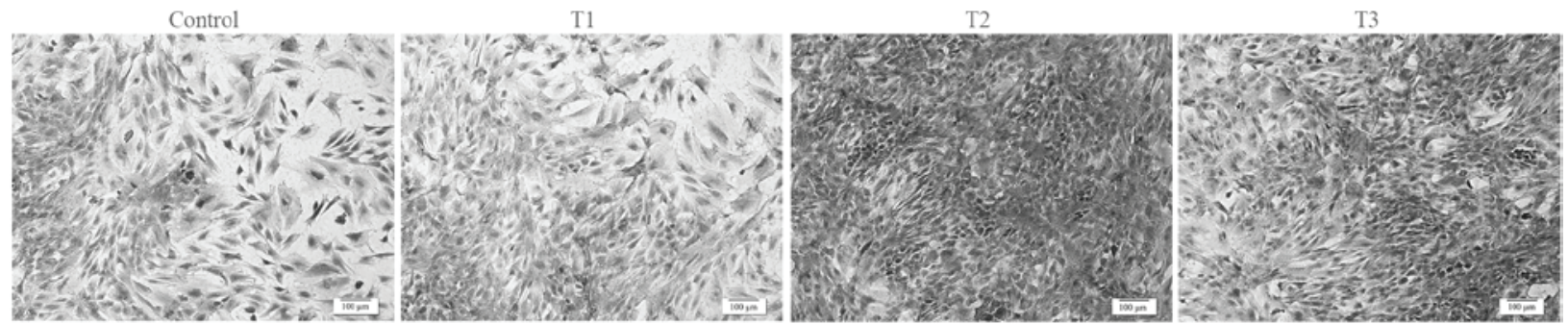

Figure 3. Hematoxylin-eosin staining images show the morphology of the chondrocytes cultured in vitro with 0 (control), 0.0625 (T1), 0.125 (T2) and $0.25 \mathrm{mmol} / 1$ (T3) protocatechuic acid for six days (cell seeding density, 2x104/ml; original magnification, x100; scale bar, $100 \mu \mathrm{m}$ ).
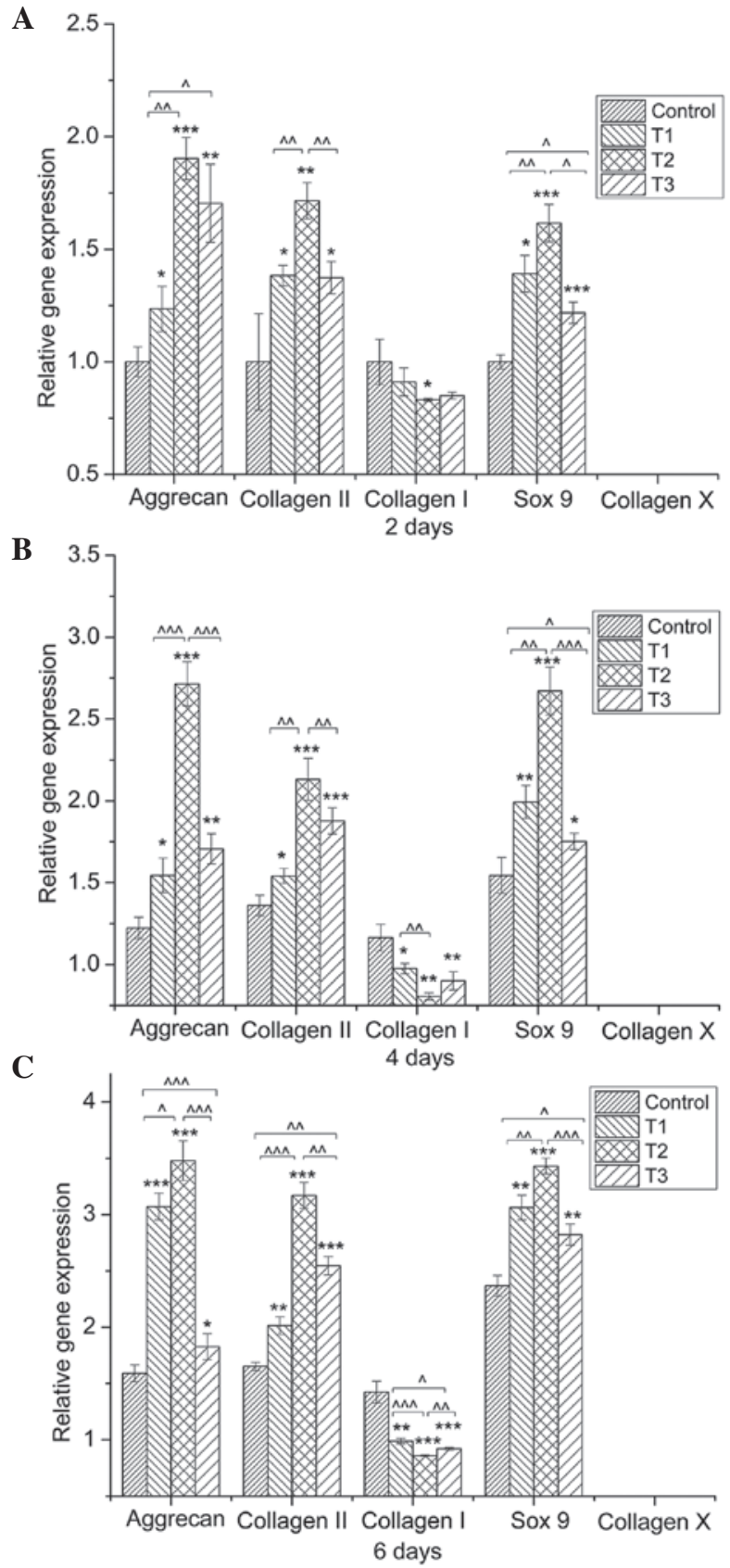

Figure 4. Quantitative comparison of extracellular-matrix-related gene expression by reverse transcription-quantitative polymerase chain reaction. Chondrocytes were cultured with 0 (control), 0.0625 (T1), 0.125 (T2) and $0.25 \mathrm{mmol} / \mathrm{l}(\mathrm{T} 3)$ protocatechuic acid (PCA) for (A) two, (B) four and (C) six days ( $\mathrm{n}=3$ for each group/time-point). Gene expression levels in the PCA groups, relative to the control group, were analyzed by the $2^{-\Delta \Delta \mathrm{Ct}}$ method using GAPDH as the internal control. Data are expressed as the mean \pm standard deviation. ${ }^{* \wedge} \mathrm{P}<0.05,{ }^{* *, \wedge} \mathrm{P}<0.01,{ }^{* * *, \wedge \wedge} \mathrm{P}<0.001$
Cell proliferation. Chondrocytes cultured with 0.0625 , 0.125 and $0.25 \mathrm{mmol} / 1 \mathrm{PCA}$ grew faster than those in the control group ( $0 \mathrm{mmol} / \mathrm{l} \mathrm{PCA})$, as indicated by the significantly higher DNA content $(\mathrm{P}<0.05)$ in the same culture period (Fig. $2 \mathrm{~A})$. Among the three concentrations, $0.125 \mathrm{mmol} / 1 \mathrm{PCA}$ exhibited the strongest promoting effect on cell growth at the same time-point of culture.

Secretion of GAGs. Fig. 2B demonstrates an evident increase in the amount of GAGs, provided as a ratio of GAG/DNA, in the PCA groups when compared with the control group for the same culture period $(\mathrm{P}<0.05)$. In line with the cell proliferation determined by DNA content, PCA at a dose of $0.125 \mathrm{mmol} / \mathrm{l}$ produced the best effect on GAG synthesis.

Cell morphology. Evaluation of chondrocyte morphology using HE staining demonstrated that chondrocytes treated with PCA grew better compared with the control group (Fig. 3). In the PCA groups, a higher number of round cells were identified, which represented the typical morphology of chondrocytes. In addition, PCA at the dose of $0.125 \mathrm{mmol} / \mathrm{l}$ was most effective at facilitating the proliferation of rabbit articular chondrocytes in vitro.

Gene expression. The positive role of PCA on ECM synthesis was further verified by examination of the expression of aggrecan, Sox9 and collagen I, II and X (Fig. 4). The mRNA expression levels of the cartilage-specific genes, aggrecan, collagen II and Sox9, were evidently promoted by PCA when compared with the control group. In addition, the expression of the collagen I gene, a marker of cell dedifferentiation, was downregulated by PCA when compared with the control cells. The expression of collagen $X$, an indicator of cell hypertrophy, was not detected. Among the experimental groups, PCA at a concentration of $0.125 \mathrm{mmol} / \mathrm{l}$ exhibited the optimum performance with regard to the upregulation of the expression levels of aggrecan, collagen II and Sox9 genes, as well as the downregulation of collagen I gene expression.

\section{Discussion}

PCA exists in numerous fruit and vegetables and is readily available in daily life. The chemical composition of PCA is similar to gallic acid, which are both categorized as a polyphenolic compounds known to exert antioxidant, anti-inflammatory and anticancer effects $(17,18)$. PCA and its analogs possess potent anti-inflammatory effects and have been 
demonstrated to be effective in animal models of arthritis (13). Based on the hypothesis that PCA may serve as a potential chondro-protective agent, the impact of PCA on the growth and phenotype maintenance of articular chondrocytes in vitro was considered in the present study. The results indicated that PCA was able to promote chondrocyte proliferation and GAG deposition in chondrocytes (Figs. 2 and 3). Proteoglycans are crucial components of the ECM (19). The ECM is responsible for maintaining the cartilage load-bearing capacity (20), and also plays a vital role in chondrocyte phenotype (21).

Consistent with the increase in GAG production, PCA was demonstrated to upregulate the gene expression of Sox9, collagen II and aggrecan (Fig. 4). The chondrogenic transcription factor, Sox 9 , is essential for increasing the rate of chondrogenesis $(22,23)$, particularly when coexpressed with collagen II (24-26). In addition, several gene therapy approaches, namely viral methods to overexpress Sox9, have been shown to significantly improve the synthesis of cartilaginous matrix produced by bone marrow-derived stem cells and articular chondrocytes (27-29). Evidence has also indicated that aggrecan production is significantly upregulated by the Sox 9 gene, as an early chondrogenic marker $(27,30)$. Therefore, a possible mechanism underlying the promotive effect of PCA on chondrocyte growth and matrix secretion may be the modulation of Sox 9 expression.

Maintaining the chondrocyte phenotype is one of the major challenges for cartilage tissue engineering and inhibition of OA development $(31,32)$. Dedifferentiation of articular chondrocytes tends to appear as the culture time progresses in tissue engineering or in the development of OA $(11,33)$. Simultaneously, non-cartilage-specific ECM is produced, which is characteristic of a poor biomechanical response. PCA has been demonstrated to enhance differentiation and benefit phenotypic survival in neural-related cells (34). In the current study, upregulation of collagen I gene expression, a marker of dedifferentiation, was not detected in the PCA groups (Fig. 4), indicating that PCA is beneficial to the phenotypic maintenance of chondrocytes. Dedifferentiation occurs when the differentiated phenotype of chondrocytes, consisting primarily of type II collagen and cartilage-specific proteoglycan, is lost and replaced by a complex collagen phenotype consisting predominately of type I collagen and a low level of proteoglycan synthesis (35-37). Hypertrophy is a predictor of secondary cell phenotype loss $(32,38)$, and the sequence of hypertrophy is endochondral ossification (39). The results of the present study revealed that the associated gene, collagen $\mathrm{X}$, was not detected in any of the groups (Fig. 4). Therefore, the reduced expression of collagen I and the undetectable expression of collagen $\mathrm{X}$ following PCA administration indicated that PCA promoted rabbit articular chondrocytes in vitro to maintain their phenotype.

As for the recommended concentration of PCA, the proliferation of rabbit articular chondrocytes in vitro was accelerated with PCA concentrations ranging between 0.05 and $0.3 \mathrm{mmol} / \mathrm{l}$. In particular, at a dose of $0.125 \mathrm{mmol} / 1$, PCA exhibited the optimum performance with regard to cell growth and phenotype maintenance. However, whether this treatment is suitable for articular chondrocytes of other species, such as humans, is unable to be confirmed. A lack of evidence also exists with regard to the application of PCA in experiments in vivo.
In conclusion, PCA exerts a positive effect on the proliferation and phenotypic maintenance of rabbit articular chondrocytes in vitro, with the optimal concentration being $0.125 \mathrm{mmol} / \mathrm{l}$. Therefore, PCA, a polyphenol compound widely found in vegetable matter, may serve as a potential agent in the field of cartilage tissue engineering and treatment of OA; however, further studies are required.

\section{Acknowledgements}

This study was supported by the National Science \& Technology Pillar Program of China (no. 2012BAI42G00), Guangxi Scientific Research and Technological Development Foundation (no. Guikehe 14125008-2-14), the Guangxi Science Fund for Distinguished Young Scholars (no. 2014GXNSFGA118006), the Key Laboratory of Regenerative Medicine of Guangxi High School and the Research Center for Regenerative Medicine and Collaborative Innovation Center of Guangxi Biological Medicine.

\section{References}

1. van der Kraan PM: Age-related alterations in TGF beta signaling as a causal factor of cartilage degeneration in osteoarthritis. Biomed Mater Eng 24 (Suppl): 75-80, 2014.

2. Tetteh ES, Bajaj S and Ghodadra NS: Basic science and surgical treatment options for articular cartilage injuries of the knee. J Orthop Sports Phys Ther 42: 243-253, 2012.

3. Stone AV, Loeser RF, Vanderman KS, Long DL, Clark SC and Ferguson CM: Pro-inflammatory stimulation of meniscus cells increases production of matrix metalloproteinases and additional catabolic factors involved in osteoarthritis pathogenesis. Osteoarthritis Cartilage 22: 264-274, 2014.

4. Ma B, Leijten JC, Wu L, et al: Gene expression profiling of dedifferentiated human articular chondrocytes in monolayer culture. Osteoarthritis Cartilage 21: 599-603, 2013.

5. Schulze-Tanzil G: Activation and dedifferentiation of chondrocytes: implications in cartilage injury and repair. Ann Anat 191: 325-338, 2009.

6. Nicolini AP, Carvalho RT, Dragone B, Lenza M, Cohen M and Ferretti M: Updates in biological therapies for knee injuries: full thickness cartilage defect. Curr Rev Musculoskelet Med 7: 256-262, 2014.

7. Drissi H, Zuscik M, Rosier R and O'Keefe R: Transcriptional regulation of chondrocyte maturation: potential involvement of transcription factors in OA pathogenesis. Mol Aspects Med 26: 169-179, 2005.

8. Bruyère $\mathrm{O}$, Cooper $\mathrm{C}$, Pelletier $\mathrm{JP}$, et al: An algorithm recommendation for the management of knee osteoarthritis in Europe and internationally: A report from a task force of the European Society for Clinical and Economic Aspects of Osteoporosis and Osteoarthritis (ESCEO). Semin Arthritis Rheum 44: 253-263, 2014.

9. Mollon B, Kandel R, Chahal J and Theodoropoulos J: The clinical status of cartilage tissue regeneration in humans. Osteoarthritis Cartilage 21: 1824-1833, 2013.

10. Fahy N, Farrell E, Ritter T, Ryan AE and Murphy JM: Immune modulation to improve tissue engineering outcomes for cartilage repair in the osteoarthritic joint. Tissue Eng Part B Rev: Aug 4, 2014 (Epub ahead of print).

11. Coates EE and Fisher JP: Phenotypic variations in chondrocyte subpopulations and their response to in vitro culture and external stimuli. Ann Biomed Eng 38: 3371-3388, 2010.

12. Ignat I, Volf I and Popa VI: A critical review of methods for characterisation of polyphenolic compounds in fruits and vegetables. Food Chem 126: 1821-1835, 2011.

13. Lende AB, Kshirsagar AD, Deshpande AD, et al: Anti-inflammatory and analgesic activity of protocatechuic acid in rats and mice. Inflammopharmacology 19: 255-263, 2011.

14. Lo CW, Huang HP, Lin HM, Chien CT and Wang CJ: Effect of Hibiscus anthocyanins-rich extract induces apoptosis of proliferating smooth muscle cell via activation of P38 MAPK and p53 pathway. Mol Nutr Food Res 51: 1452-1460, 2007. 
15. Yoon CH, Chung SJ, Lee SW, Park YB, Lee SK and Park MC: Gallic acid, a natural polyphenolic acid, induces apoptosis and inhibits proinflammatory gene expressions in rheumatoid arthritis fibroblast-like synoviocytes. Joint Bone Spine 80: 274-279, 2013.

16. GuanS,Zhang XL,GeD,LiuTQ,MaXH and CuiZF: Protocatechuic acid promotes the neuronal differentiation and facilitates survival of phenotypes differentiated from cultured neural stem and progenitor cells. Eur J Pharmacol 670: 471-478, 2011.

17. Kakkar S and Bais S: A review on protocatechuic acid and its pharmacological potential. ISRN Pharmacol 2014: 952943, 2014

18. Hsu CC, Hsu CL, Tsai SE, Fu TY and Yen GC: Protective effect of Millettia reticulata Benth against $\mathrm{CCl}(4)$-induced hepatic damage and inflammatory action in rats. J Med Food 12: 821-828, 2009.

19. Buschmann MD and Grodzinsky AJ: A molecular model of proteoglycan-associated electrostatic forces in cartilage mechanics. J Biomech Eng 117: 179-192, 1995.

20. Horkay F: Interactions of cartilage extracellular matrix macromolecules. J Polym Sci B Polym Phys 50: 1699-1705, 2012.

21. Grogan SP, Chen X, Sovani S, et al: Influence of cartilage extracellular matrix molecules on cell phenotype and neocartilage formation. Tissue Eng Part A 20: 264-274, 2014.

22. Akiyama H: Transcriptional regulation in chondrogenesis by Sox9. Clin Calcium 21: 845-851, 2011 (In Japanese)

23. Tew SR and Clegg PD: Analysis of post transcriptional regulation of SOX9 mRNA during in vitro chondrogenesis. Tissue Eng Part A 17: 1801-1807, 2011.

24. Ng LJ, Wheatley S, Muscat GE, et al: SOX9 binds DNA, activates transcription, and coexpresses with type II collagen during chondrogenesis in the mouse. Dev Biol 183: 108-121, 1997.

25. Marshall OJ and Harley VR: Molecular mechanisms of SOX9 action. Mol Genet Metab 71: 455-462, 2000.

26. Davies SR, Chang LW, Patra D, et al: Computational identification and functional validation of regulatory motifs in cartilage-expressed genes. Genome Res 17: 1438-1447, 2007.

27. Tew SR, Li Y, Pothacharoen P, Tweats LM, Hawkins RE and Hardingham TE: Retroviral transduction with SOX9 enhances re-expression of the chondrocyte phenotype in passaged osteoarthritic human articular chondrocytes. Osteoarthritis Cartilage 13 80-89, 2005.
28. Paul R, Haydon RC, Cheng H, et al: Potential use of Sox9 gene therapy for intervertebral degenerative disc disease. Spine 28: 755-763, 2003.

29. Tsuchiya H, Kitoh H, Sugiura F and Ishiguro N: Chondrogenesis enhanced by overexpression of sox 9 gene in mouse bone marrow-derived mesenchymal stem cells. Biochem Biophys Res Commun 301: 338-343, 2003

30. Bi W, Deng JM, Zhang Z, Behringer RR and de Crombrugghe B: Sox9 is required for cartilage formation. Nat Genet 22: 85-89, 1999.

31. Gan L and Kandel RA: In vitro cartilage tissue formation by co-culture of primary and passaged chondrocytes. Tissue Eng 13 831-842, 2007.

32. Fosang AJ and Beier F: Emerging frontiers in cartilage and chondrocyte biology. Best Pract Res Clin Rheumatol 25: 751-766, 2011.

33. Bailey AM: Balancing tissue and tumor formation in regenerative medicine. Sci Transl Med 4: 147fs 28, 2012

34. Guan S, Ge D, Liu TQ, Ma XH and Cui ZF: Protocatechuic acid promotes cell proliferation and reduces basal apoptosis in cultured neural stem cells. Toxicol In Vitro 23: 201-208, 2009.

35. Benya PD and Shaffer JD: Dedifferentiated chondrocytes reexpress the differentiated collagen phenotype when cultured in agarose gels. Cell 30: 215-224, 1982.

36. Schnabel M, Marlovits S, Eckhoff G, et al Dedifferentiation-associated changes in morphology and gene expression in primary human articular chondrocytes in cell culture. Osteoarthritis Cartilage 10: 62-70, 2002.

37. Karlsen TA, Shahdadfar A and Brinchmann JE: Human primary articular chondrocytes, chondroblasts-like cells, and dedifferentiated chondrocytes: differences in gene, microRNA, and protein expression and phenotype. Tissue Eng Part C Methods 17: 219-227, 2011.

38. Grassel S and Ahmed N: Influence of cellular microenvironment and paracrine signals on chondrogenic differentiation. Front Biosci 12: 4946-4956, 2007.

39. Dreier R: Hypertrophic differentiation of chondrocytes in osteoarthritis: the developmental aspect of degenerative joint disorders. Arthritis Res Ther 12: 216, 2010. 